\title{
A $\delta$-Invariant for QR-Submanifolds in Quaternion Space Forms
}

\author{
Gabriel Macsim and Adela Mihai*
}

(Communicated by Cihan Özgür)

\begin{abstract}
Starting from an inequality involving the invariant $\delta(\mathcal{D})$ for an anti-holomorphic submanifold of a complex space form [1] and using optimization methods on Riemannian manifolds, we establish a corresponding inequality for the invariant $\delta\left(\mathcal{D}^{\perp}\right)$ defined on QR-submanifolds in quaternion space forms, in terms of the squared mean curvature. We obtain a relationship between intrinsic and extrinsic invariants for QR-submanifolds of quaternion space forms.
\end{abstract}

Keywords: QR-submanifolds, quaternion space forms, $\delta$-invariants.

AMS Subject Classification (2010): Primary: 53C40; Secondary: 53C25.

\section{Introduction}

The fact that every Riemannian manifold can be regarded as a Riemannian submanifold isometrically embedded in some Euclidean space with sufficiently high codimension (according to the embedding theorem of J.F. Nash [6]) gives the opportunity to use the extrinsic help in Riemannian geometry. One of the most fundamental problems in the theory of submanifold is to find simple relationships between intrinsic and extrinsic invariants of a submanifold.

In this paper, we consider $\delta$-invariants of QR-submanifolds of a quaternion space forms; they are very important among intrinsic invariants, being different in nature from the classical Ricci and scalar curvature. The non-trivial $\delta$-invariants are obtained from scalar curvature by substracting a certain amount of sectional curvatures.

Let $\tilde{M}$ be a Kaehler manifold with complex structure $J$ and let $N$ be a Riemannian manifold isometrically immersed in $\tilde{M}$. One denotes by $\mathcal{D}_{x}, x \in N$, the maximal complex subspace $T_{x} N \cap J\left(T_{x} N\right)$ of the tangent space $T_{x} N$ of $N$. If the dimension of $\mathcal{D}_{x}$ is constant for all $x \in N$, then $\mathcal{D}: x \rightarrow \mathcal{D}_{x}$ defines a holomorphic distribution $\mathcal{D}$ on $N$. A subspace $\nu$ of $T_{x} N, x \in N$, is called totally real if $J(\nu)$ is a subspace of the normal space $T_{x}^{\perp} N$ at $x$. If each tangent space of $N$ is totally real, then $N$ is called a totally real submanifold of the Kaehler manifold $\tilde{M}$.

If the orthogonal complementary distribution $\mathcal{D}^{\perp}$ of the holomorphic distribution $\mathcal{D}$ is totally real, i.e., $T N=\mathcal{D} \oplus \mathcal{D}^{\perp}, J \mathcal{D}_{x}^{\perp} \subset T_{x}^{\perp} N, x \in N$, then the submanifold $N$ is called a $C R$-submanifold. ([3]).

The totally real distribution $\mathcal{D}^{\perp}$ of every $C R$-submanifold of a Kaehler manifold is an integrable distribution

In order to give some answers to an open question concerning minimal immersions proposed by S. S. Chern in the 1960's and to provide some applications of the Nash embedding theorem, B.-Y. Chen introduced in early 1990 's the notion of $\delta$-invariants. In the case of a $C R$-submanifold $N$ of a Kaehler manifold, Chen introduced two $\delta$-invariants $\delta(\mathcal{D})$ and $\delta\left(\mathcal{D}^{\perp}\right)$, called $C R \delta$-invariants, defined by Chen in [4]:

$$
\begin{gathered}
\delta(\mathcal{D})(x)=\tau(x)-\tau\left(\mathcal{D}_{x}\right), \\
\delta\left(\mathcal{D}^{\perp}\right)(x)=\tau(x)-\tau\left(\mathcal{D}_{x}^{\perp}\right),
\end{gathered}
$$

where $\tau$ is the scalar curvature of $N$ and $\tau\left(\mathcal{D}_{x}\right)$ and $\tau\left(\mathcal{D}^{\perp}\right)$ are the scalar curvature of the holomorphic distribution $\mathcal{D}$ and totally real distribution $\mathcal{D}^{\perp}$ of $N$, respectively. 
In [1], Al-Solamy, Chen and Deshmukh proved an inequality involving the $\delta$-invariant $\delta(\mathcal{D})$, for an antiholomorphic submanifold in a complex space form, in terms of the squared mean curvature.

In 1986, A. Bejancu [2] introduced the notion of $Q R$-submanifolds as a generalization of real hypersurfaces of a quaternion Kaehler manifold (see also [8]).

Let $\tilde{M}$ be a quaternion Kaehler manifold and $N$ be a real submanifold of $\tilde{M} . N$ is called a $Q R$-submanifold if there exists a vector subbundle $\nu$ of the normal bundle such that we have

$$
J_{\alpha}\left(\nu_{x}\right)=\nu_{x} \text { and } J_{\alpha}\left(\nu_{x}^{\perp}\right) \subset T_{x} N, x \in N, \alpha=\overline{1,3},
$$

where $\nu^{\perp}$ is the complementary orthogonal bundle.

Taking into account the research done until now ([5]), we remark that quaternion $C R$-submanifolds and $Q R$-submanifolds have very little in common (see also section 2).

In the present paper, we give a corresponding inequality to the inequality given in [1], for $\delta\left(\mathcal{D}^{\perp}\right)$ in the case of a QR-submanifold of a quaternion space form with minimal codimension, i.e., $\operatorname{dim} \nu_{x}=0$.

\section{Basics on quaternion manifolds and submanifolds}

Let $\tilde{M}$ be a Riemannian manifold and $N \subset \tilde{M}$ a Riemannian submanifold of $\tilde{M}$ with the induced Riemannian metric. We denote by $T N$ and $T^{\perp} N$ the tangent bundle, respectively the normal bundle of $N$, and by $\nabla$ and $\tilde{\nabla}$ the Levi-Civita connections of $N$ and $\tilde{M}$, respectively.

The Gauss and Weingarten formulae are given by:

$$
\begin{gathered}
\tilde{\nabla}_{X} Y=\nabla_{X} Y+h(X, Y), \\
\tilde{\nabla}_{X} V=-A_{V} X+\nabla_{X}^{\perp} V,
\end{gathered}
$$

$\forall X, Y \in \Gamma(T N), V \in \Gamma\left(T^{\perp} N\right)$, where $\nabla^{\perp}$ is the normal connection on $T^{\perp} N$.

One has $g(h(X, Y), V)=g\left(A_{V} X, Y\right)$.

If $\tilde{M}$ is a $4 n$-dimensional manifold with the Riemannian metric $g$, then $\tilde{M}$ is called a quaternion Kaehler manifold if there exists a 3-dimensional vector bundle $\sigma$ of local basis of almost Hermitian structures $J_{1}, J_{2}, J_{3}$ such that

$$
J_{\alpha} \circ J_{\alpha+1}=-J_{\alpha+1} \circ J_{\alpha}=J_{\alpha+2}, J_{\alpha}^{2}=-\mathrm{Id},
$$

where $\alpha, \alpha+1, \alpha+2$ are taken modulo 3 .

In this case, $\sigma$ is called an almost quaternion structure on $\tilde{M},\left\{J_{1}, J_{2}, J_{3}\right\}$ is the canonical local basis of $\sigma$ and $(\tilde{M}, \sigma)$ is called an almost quaternion manifold, with $\operatorname{dim} \tilde{M}=4 m, m \geq 1$.

A Riemannian metric $\tilde{g}$ on $\tilde{M}$ is said to be adapted to the almost quaternion structure $\sigma$ if it satisfies

$$
\tilde{g}\left(J_{\alpha} X, J_{\alpha} Y\right)=\tilde{g}(X, Y), \forall \alpha=\overline{1,3} .
$$

Then $(\tilde{M}, \sigma, \tilde{g})$ is called an almost quaternion Hermitian manifold.

If $\sigma$ is parallel with respect to $\tilde{\nabla}$, then $(\tilde{M}, \sigma, \tilde{g})$ is called a quaternion Kaehler manifold. Equivalently, there exist locally defined 1-forms $\omega_{1}, \omega_{2}, \omega_{3}$ such that $\forall \alpha=\overline{1,3},\left(\tilde{\nabla}_{X} J_{\alpha}\right)(X)=\omega_{\alpha+2}(X) J_{\alpha+1}-\omega_{\alpha+1} J_{\alpha+2}$, where $\alpha, \alpha+1, \alpha+2$ are taken modulo 3 .

Remark 2.1. Any quaternion Kaehler manifold is an Einstein manifold (for $\operatorname{dim} \tilde{M} \geq 4$ ).

Let $(\tilde{M}, \sigma, \tilde{g})$ be a quaternion Kaehler manifold and $X$ be a non-null vector on $\tilde{M}$. Then the 4-plane spanned by $\left\{X, J_{1} X, J_{2} X, J_{3} X\right\}$, denoted by $Q(X)$, is called a quaternion 4-plane. Any 2-plane in $Q(X)$ is called a quaternion plane. The sectional curvature of a quaternion plane is called a quaternion sectional curvature.

A quaternion Kaehler manifold is called a quaternion space form if its quaternion sectional curvature is constant, say c. So, $(\tilde{M}, \sigma, \tilde{g})$ is a quaternion space form if and only if

$$
\begin{gathered}
\tilde{R}(X, Y) Z=\frac{c}{4}\{\tilde{g}(Y, Z) X-\tilde{g}(X, Z) Y+ \\
\left.+\sum_{\alpha=1}^{3}\left[\tilde{g}\left(Z, J_{\alpha} Y\right) J_{\alpha} X-\tilde{g}\left(Z, J_{\alpha} X\right) J_{\alpha} Y+2 \tilde{g}\left(X, J_{\alpha} Y\right) J_{\alpha} Z\right]\right\}
\end{gathered}
$$


$\forall X, Y, Z \in \Gamma(T \tilde{M})$.

For a submanifold $N$ of $\tilde{M}$, if $\left\{e_{1}, \ldots, e_{n}\right\}$ is an orthonormal basis of $T_{p} N$ and $\left\{e_{n+1}, \ldots, e_{4 m}\right\}$ an orthonormal basis of $T_{p}^{\perp} N, p \in N$, the mean curvature vector is given by

$$
H(p)=\frac{1}{n} \sum_{i=1}^{n} h\left(e_{i}, e_{i}\right)
$$

One denotes by

$$
\|h\|^{2}(p)=\sum_{i, j=1}^{n} g\left(h\left(e_{i}, e_{j}\right), h\left(e_{i}, e_{j}\right)\right) .
$$

For a quaternion Kaehler manifold, we have

$$
\left(\tilde{\nabla}_{X} J_{\alpha}\right)(X)=\sum_{\beta=1}^{3} Q_{\alpha \beta}(X) J_{\beta}, \alpha=\overline{1,3}, \forall X \in \Gamma(T \tilde{M}),
$$

where $Q_{\alpha \beta}$ are certain 1-forms locally defined on $\tilde{M}$ such that $Q_{\alpha \beta}+Q_{\beta \alpha}=0$.

Let $\tilde{M}$ be a quaternion Kaehler manifold and $N$ be a real submanifold of $\tilde{M} . N$ is called a $Q R$-submanifold if there exists a vector subbundle $\nu$ of the normal bundle such that

$$
J_{\alpha}\left(\nu_{x}\right)=\nu_{x} \text { and } J_{\alpha}\left(\nu_{x}^{\perp}\right) \subset T_{x} N, x \in N, \alpha=\overline{1,3},
$$

where $\nu^{\perp}$ is the complementary orthogonal bundle.

Let $\mathcal{D}_{\alpha x}=J_{\alpha}\left(\nu_{x}^{\perp}\right), \mathcal{D}_{x}^{\perp}=\mathcal{D}_{1 x} \oplus \mathcal{D}_{2 x} \oplus \mathcal{D}_{3 x}$ a 3q-dimensional distribution $\mathcal{D}^{\perp}: x \rightarrow \mathcal{D}_{x}^{\perp}$ globally defined on $N$, where $q=\operatorname{dim} \nu_{x}^{\perp}$. One has

$$
J_{\alpha}\left(\mathcal{D}_{\alpha x}\right)=\nu_{x}^{\perp}, J_{\alpha}\left(\mathcal{D}_{\beta x}\right)=\mathcal{D}_{\gamma x}, \forall x \in N,
$$

where $(\alpha, \beta, \gamma)$ is a cyclic permutation of $(1,2,3)$.

$\mathcal{D}$ is the orthogonal complementary distribution of $\mathcal{D}^{\perp}$ in $T N$ and $J_{\alpha}\left(\mathcal{D}_{x}\right)=\mathcal{D}_{x} . \mathcal{D}$ is called the quaternion distribution.

So

$$
\begin{gathered}
T \tilde{M}=T N \oplus T^{\perp} N, T N=\mathcal{D} \oplus \mathcal{D}^{\perp}, \\
T^{\perp} N=\nu \oplus \nu^{\perp}, \nu, \nu^{\perp} \subset T^{\perp} N, \mathcal{D}_{x}^{\perp}=\mathcal{D}_{1 x} \oplus \mathcal{D}_{2 x} \oplus \mathcal{D}_{3 x} .
\end{gathered}
$$

Recall that $N$ is a quaternion $C R$-submanifold (see [5]) if it admits a differential quaternion distribution $\mathcal{D}$ such that its orthogonal complementary distribution $\mathcal{D}^{\perp}$ is totally real, i.e. $J_{\alpha}\left(\mathcal{D}_{x}^{\perp}\right) \subset T_{x}^{\perp} N$, for $\alpha=1,2,3$ and $\forall x \in N$.

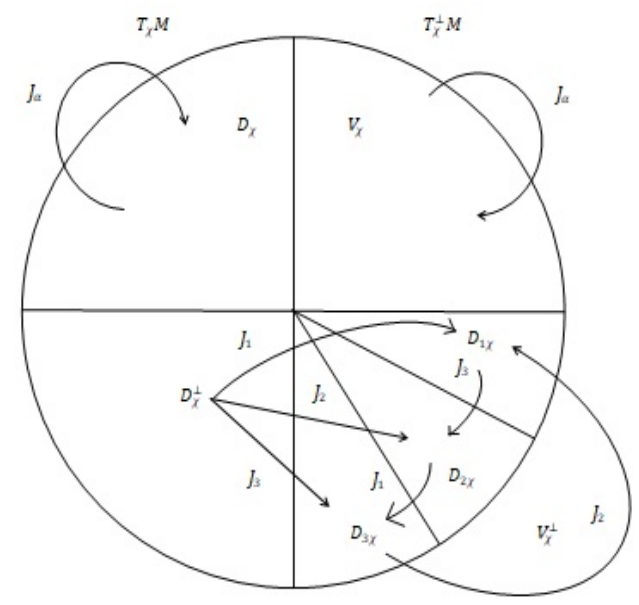

Figure 1. Quaternion CR-submanifolds 


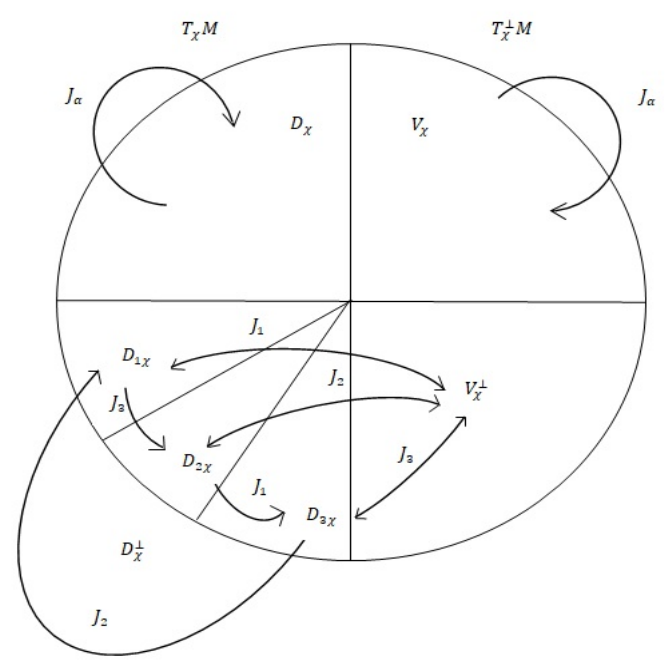

Figure 2. QR-submanifolds

The differences between the quaternion CR-submanifolds and QR-submanifolds in quaternion space forms can be represented in the Figure 1 and Figure 2.

For $Y \in \Gamma(T N)$ we consider the decomposition $J_{\alpha} Y=\Phi_{\alpha} Y+F_{\alpha} Y, \alpha=\overline{1,3} ; \Phi_{\alpha} Y, F_{\alpha} Y$ are the tangential and normal components of $J_{\alpha} Y$, respectively.

For $V \in \Gamma\left(T^{\perp} N\right)$ we consider the decomposition $J_{\alpha} V=t_{\alpha} V+f_{\alpha} V, \alpha=\overline{1,3} ; t_{\alpha} V, f_{\alpha} V$ are the tangential and normal components of $J_{\alpha} V$, respectively.

$N$ is called mixed geodesic if $h(X, Y)=0, \forall X \in \Gamma(\mathcal{D}), Y \in \Gamma\left(\mathcal{D}^{\perp}\right)$.

Let $\pi=\operatorname{sp}\{X, Y\}$ be a tangent plane to $\tilde{M}$ at a point $p \in \tilde{M}$. The sectional curvature of $\pi$ is

$$
\tilde{K}(\pi)=\frac{\tilde{R}(X, Y, X, Y)}{\tilde{g}(X, X) \tilde{g}(Y, Y)-\tilde{g}^{2}(X, Y)} .
$$

From

$$
\begin{gathered}
\tilde{R}(X, Y) Z=\frac{c}{4}\{\tilde{g}(Z, Y) X-\tilde{g}(X, Z) Y+ \\
\left.+\sum_{\alpha=1}^{3}\left[\tilde{g}\left(Z, J_{\alpha} Y\right) J_{\alpha} X-\tilde{g}\left(Z, J_{\alpha} X\right) J_{\alpha} Y+2 \tilde{g}\left(X, J_{\alpha} Y\right) J_{\alpha} Z\right]\right\}
\end{gathered}
$$

we obtain

$$
\tilde{K}(X \wedge Y)=\frac{c}{4}\left[1+3 \sum_{\alpha=1}^{3} \tilde{g}^{2}\left(J_{\alpha} X, Y\right)\right],
$$

$\forall X, Y \in \Gamma\left(T_{p} \tilde{M}\right), p \in \tilde{M}$, unit vector fields.

By the Gauss equation, we have

$$
K(X \wedge Y)=\frac{c}{4}\left[1+3 \sum_{\alpha=1}^{3} \tilde{g}^{2}\left(J_{\alpha} X, Y\right)\right]+\tilde{g}(h(X, X), h(Y, Y))-\tilde{g}(h(X, Y), h(X, Y)) .
$$

We recall the following result.

Let $(N, g)$ be a Riemannian submanifold of a Riemannian manifold $(\tilde{M}, \tilde{g})$ and $f \in \mathcal{C}^{\infty}(\tilde{M})$. We attach the following Optimum Problem:

$$
\min _{x \in N} f(x)
$$


Theorem 2.1. [7] If $x_{0} \in N$ is a solution of the problem (2.1), then

a) $(\operatorname{grad} f)\left(x_{0}\right) \in T_{x_{0}}^{\perp} N$

b) the bilinear form $\beta: T_{x_{0}} N \times T_{x_{0}} N \rightarrow \mathbb{R}$,

$$
\beta(X, Y)=\operatorname{Hess}_{f}(X, Y)+\tilde{g}\left(h(X, Y),(\operatorname{grad} f)\left(x_{0}\right)\right)
$$

is positive semidefinite, where $h$ is the second fundamental form of the submanifold $N$ in $\tilde{M}$.

Remark 2.2. If $\beta$ is negative semidefinite, then we have a solution of $\max _{x \in N} f(x)$.

\section{An inequality for a new $\delta$-invariant}

If $N \subset \tilde{M}$ is a QR-submanifold of minimal codimension, i.e., $\operatorname{dim} \nu_{x}=0$ for $x \in M$, we consider the following orthonormal bases:

$$
\begin{gathered}
\left\{e_{1}, \ldots, e_{n}\right\} \subset \mathcal{D}_{x} ; \\
\left\{J_{1} e_{n+1}, \ldots, J_{1} e_{n+q} ; J_{2} e_{n+1}, \ldots, J_{2} e_{n+q} ; J_{3} e_{n+1}, \ldots, J_{3} e_{n+q}\right\} \subset \mathcal{D}_{x}^{\perp} ; \\
\left\{e_{n+1}, \ldots, e_{n+q}\right\} \subset T_{x}^{\perp} N .
\end{gathered}
$$

For $x \in N$, we have

$$
\begin{gathered}
\operatorname{dim} \mathcal{D}_{x}=n ; \operatorname{dim} \mathcal{D}_{x}^{\perp}=3 q ; \operatorname{dim} T_{x} N=n+3 q ; \\
\operatorname{dim} \nu_{x}=0, \operatorname{dim} T_{x}^{\perp} N=q=\operatorname{dim} \nu_{x}^{\perp} .
\end{gathered}
$$

We define the following $\mathrm{QR} \delta$-invariant $\delta\left(\mathcal{D}^{\perp}\right)$ by

$$
\delta\left(\mathcal{D}^{\perp}\right)(x)=\tau(x)-\tau\left(\mathcal{D}_{x}^{\perp}\right), x \in \tilde{M},
$$

where $\tau$ and $\tau\left(\mathcal{D}^{\perp}\right)$ denote the scalar curvature of $N$ and the scalar curvature of the distribution $\mathcal{D}^{\perp} \subset T N$, respectively.

In the following, we will use the convention on range of indices, unless mentioned otherwise:

$$
i, j, k=\overline{1, n} ; \alpha, \beta, \gamma=\overline{1,3} ; r, s, t=\overline{n+1, n+q} ; A, B, C=\overline{1, n+q} .
$$

In [1], the authors proved an inequality for $\delta(\mathcal{D})$ for an anti-holomorphic submanifold of a complex space form:

Theorem 3.1. [1] Let $N$ be an anti-holomorphic submanifold of a complex space form $\tilde{M}^{h+p}(c)$ with $h=\operatorname{rank}_{\mathbf{C}} \mathcal{D} \geq 1$ and $p=\operatorname{rank} \mathcal{D}^{\perp} \geq 2$. Then we have

$$
\delta(\mathcal{D}) \leq \frac{(p-1)(2 h+p)^{2}}{2(p+2)} \cdot\|H\|^{2}+\frac{p}{2}(4 h+p-1) \cdot \frac{c}{4} .
$$

The equality sign holds identically if and only if the following three conditions are satisfied:

(a) $N$ is D-minimal,

(b) $N$ is mixed geodesic, and

(c) there exists an orthonormal frame $\left\{e_{2 h+1}, \ldots, e_{n}\right\}$ of $\mathcal{D}^{\perp}$ such that the second fundamental form $\sigma$ of $N$ satisfies

$\sigma_{r r}^{r}=3 \sigma_{s s}^{r}$, for $2 h+1 \leq r \neq s \leq 2 h+p$, and

$\sigma_{r s}^{t}=0$ for distinct $r, s, t \in\{2 h+1, \ldots, 2 h+p\}$.

The main result of our study is the following inequality involving $\delta\left(\mathcal{D}^{\perp}\right)$ for a QR-submanifold of a quaternion space form.

Theorem 3.2. Let $N$ be a QR-submanifold of minimal codimension of a quaternion space form $\tilde{M}(c), \operatorname{dim} \mathcal{D}_{x}=$ $n, \operatorname{dim} \mathcal{D}_{x}^{\perp}=3 q, \operatorname{dim} \nu_{x}=0, \operatorname{dim} \nu_{x}^{\perp}=q, x \in N$. Then we have:

$$
\delta\left(\mathcal{D}^{\perp}\right) \leq \frac{n(n+3 q)^{2}}{2(n+1)} \cdot\|H\|^{2}+\frac{n(n+6 q+8)}{2} \cdot \frac{c}{4} .
$$


The equality sign holds identically if and only if the following three conditions are satisfied:

(a) $N$ is mixed geodesic,

(b) the distribution $\mathcal{D}$ is totally umbilical, and

(c) there exists an orthonormal frame

$$
\left\{J_{1} e_{n+1}, \ldots, J_{1} e_{n+q} ; J_{2} e_{n+1}, \ldots, J_{2} e_{n+q} ; J_{3} e_{n+1}, \ldots, J_{3} e_{n+q}\right\}
$$

of $\mathcal{D}_{x}^{\perp}$ such that the second fundamental form $\sigma$ of $N$ satisfies

$$
h_{i j}^{r}=0, i, j=\overline{1, n}, i \neq j, r=\overline{n+1, n+q} .
$$

Proof. With the above notations, for $x \in N$ we have

$$
\begin{gathered}
\tau(x)=\sum_{1 \leq i<j \leq n} K\left(e_{i} \wedge e_{j}\right)+\sum_{\alpha, \beta=1}^{3} \sum_{r, s=n+1}^{n+q} K\left(J_{\alpha} e_{r} \wedge J_{\beta} e_{s}\right)+\sum_{\alpha=1}^{3} \sum_{i=1}^{n} \sum_{r=n+1}^{n+q} K\left(e_{i} \wedge J_{\alpha} e_{r}\right) . \\
\tau\left(\mathcal{D}_{x}^{\perp}\right)=\sum_{\alpha, \beta=1}^{3} \sum_{r, s=n+1}^{n+q} K\left(J_{\alpha} e_{r} \wedge J_{\beta} e_{s}\right) .
\end{gathered}
$$

From these two relations, we obtain

$$
\delta\left(\mathcal{D}^{\perp}\right)(x)=\sum_{1 \leq i<j \leq n} K\left(e_{i} \wedge e_{j}\right)+\sum_{\alpha=1}^{3} \sum_{i=1}^{n} \sum_{r=n+1}^{n+q} K\left(e_{i} \wedge J_{\alpha} e_{r}\right) .
$$

Applying the Gauss equation for $X=e_{i}, Y=e_{j}, i, j=\overline{1, n}, i \neq j$, we get

$$
K\left(e_{i} \wedge e_{j}\right)=\frac{c}{4}\left[1+3 \sum_{\alpha=1}^{3} \tilde{g}^{2}\left(J_{\alpha} e_{i}, e_{j}\right)\right]+\tilde{g}\left(h\left(e_{i}, e_{i}\right), h\left(e_{j}, e_{j}\right)\right)-\tilde{g}\left(h\left(e_{i}, e_{j}\right), h\left(e_{i}, e_{j}\right)\right) .
$$

Because $J_{\alpha} e_{i} \in \mathcal{D}$ and $J_{\alpha} e_{r} \in \mathcal{D}^{\perp}$, by applying the Gauss equation for $X=e_{i}$ and $Y=J_{\alpha} e_{r}, i=\overline{1, n}, r=$ $\overline{n+1, n+q}, \alpha=\overline{1,3}$, we have

$$
K\left(e_{i} \wedge J_{\alpha} e_{r}\right)=\frac{c}{4}+\tilde{g}\left(h\left(e_{i}, e_{i}\right), h\left(J_{\alpha} e_{r}, J_{\alpha} e_{r}\right)\right)-\tilde{g}\left(h\left(e_{i}, J_{\alpha} e_{r}\right), h\left(e_{i}, J_{\alpha} e_{r}\right)\right) .
$$

Using the relations (3.2) and (3.3) in (3.1), it follows that

$$
\begin{gathered}
\delta\left(\mathcal{D}^{\perp}\right)(x)=\sum_{1 \leq i<j \leq n}\left\{\frac{c}{4}\left[1+3 \sum_{\alpha=1}^{3} \tilde{g}^{2}\left(J_{\alpha} e_{i}, e_{j}\right)\right]+\tilde{g}\left(h\left(e_{i}, e_{i}\right), h\left(e_{j}, e_{j}\right)\right)-\tilde{g}\left(h\left(e_{i}, e_{j}\right), h\left(e_{i}, e_{j}\right)\right)\right\}+ \\
+\sum_{\alpha=1}^{3} \sum_{i=1}^{n} \sum_{r=n+1}^{n+q}\left[\frac{c}{4}+\tilde{g}\left(h\left(e_{i}, e_{i}\right), h\left(J_{\alpha} e_{r}, J_{\alpha} e_{r}\right)\right)-\tilde{g}\left(h\left(e_{i}, J_{\alpha} e_{r}\right), h\left(e_{i}, J_{\alpha} e_{r}\right)\right)\right]= \\
=\frac{n(n-1)}{2} \cdot \frac{c}{4}+\frac{3 c}{4} \sum_{\alpha=1}^{3} \sum_{1 \leq i<j \leq n} \tilde{g}^{2}\left(J_{\alpha} e_{i}, e_{j}\right)+ \\
+\sum_{1 \leq i<j \leq n}\left[\tilde{g}\left(h\left(e_{i}, e_{i}\right), h\left(e_{j}, e_{j}\right)\right)-\tilde{g}\left(h\left(e_{i}, e_{j}\right), h\left(e_{i}, e_{j}\right)\right)\right]+ \\
+3 n q \cdot \frac{c}{4}+\sum_{\alpha=1}^{3} \sum_{i=1}^{n} \sum_{r=n+1}^{n+q}\left[\tilde{g}\left(h\left(e_{i}, e_{i}\right), h\left(J_{\alpha} e_{r}, J_{\alpha} e_{r}\right)\right)-\tilde{g}\left(h\left(e_{i}, J_{\alpha} e_{r}\right), h\left(e_{i}, J_{\alpha} e_{r}\right)\right)\right] ;
\end{gathered}
$$

thus we obtain

$$
\delta\left(\mathcal{D}^{\perp}\right)(x)=\frac{n(n+6 q-1)}{2} \cdot \frac{c}{4}+\frac{3 c}{4} \sum_{\alpha=1}^{3} \sum_{1 \leq i<j \leq n} \tilde{g}^{2}\left(J_{\alpha} e_{i}, e_{j}\right)+
$$




$$
\begin{aligned}
& +\sum_{1 \leq i<j \leq n} \tilde{g}\left(h\left(e_{i}, e_{i}\right), h\left(e_{j}, e_{j}\right)\right)+\sum_{\alpha=1}^{3} \sum_{i=1}^{n} \sum_{r=n+1}^{n+q} \tilde{g}\left(h\left(e_{i}, e_{i}\right), h\left(J_{\alpha} e_{r}, J_{\alpha} e_{r}\right)\right)- \\
& -\sum_{1 \leq i<j \leq n} \tilde{g}\left(h\left(e_{i}, e_{j}\right), h\left(e_{i}, e_{j}\right)\right)-\sum_{\alpha=1}^{3} \sum_{i=1}^{n} \sum_{r=n+1}^{n+q} \tilde{g}\left(h\left(e_{i}, J_{\alpha} e_{r}\right), h\left(e_{i}, J_{\alpha} e_{r}\right)\right) .
\end{aligned}
$$

Obviously

$$
\left\|P_{\alpha}\right\|^{2}=\sum_{i, j=1}^{n} \tilde{g}^{2}\left(J_{\alpha} e_{i}, e_{j}\right)=n .
$$

Taking into account that the term $\sum_{\alpha=1}^{3} \sum_{i=1}^{n} \sum_{r=n+1}^{n+q} \tilde{g}\left(h\left(e_{i}, J_{\alpha} e_{r}\right), h\left(e_{i}, J_{\alpha} e_{r}\right)\right)$ is positive (being a sum of squares), the relations (3.4) and (3.5) imply

$$
\begin{gathered}
\delta\left(\mathcal{D}^{\perp}\right)(x) \leq \frac{n(n+6 q-1)}{2} \cdot \frac{c}{4}+9 n \frac{c}{8}+\sum_{1 \leq i<j \leq n} \tilde{g}\left(h\left(e_{i}, e_{i}\right), h\left(e_{j}, e_{j}\right)\right)+ \\
+\sum_{\alpha=1}^{3} \sum_{i=1}^{n} \sum_{r=n+1}^{n+q} \tilde{g}\left(h\left(e_{i}, e_{i}\right), h\left(J_{\alpha} e_{r}, J_{\alpha} e_{r}\right)\right)-\sum_{1 \leq i<j \leq n} \tilde{g}\left(h\left(e_{i}, e_{j}\right), h\left(e_{i}, e_{j}\right)\right)= \\
=\frac{n(n+6 q+8)}{2} \cdot \frac{c}{4}+\sum_{1 \leq i<j \leq n} \sum_{r=n+1}^{n+q} h_{i i}^{r} h_{j j}^{r}+ \\
+\sum_{i=1}^{n} \sum_{r, s=n+1}^{n+q} h_{i i}^{s}\left[\tilde{h}_{r r}^{s}+\tilde{\tilde{h}}_{r r}^{s}+\tilde{\tilde{h}}_{r r}^{s}\right]-\sum_{1 \leq i<j \leq n} \sum_{r=n+1}^{n+q}\left(h_{i j}^{r}\right)^{2},
\end{gathered}
$$

where

$$
\begin{gathered}
h_{i j}^{r}=\tilde{g}\left(h\left(e_{i}, e_{j}\right), e_{r}\right), \\
\tilde{h}_{r s}^{t}=\tilde{g}\left(h\left(J_{1} e_{r}, J_{1} e_{s}\right), e_{t}\right), \\
\tilde{\tilde{h}}_{r s}^{t}=\tilde{g}\left(h\left(J_{2} e_{r}, J_{2} e_{s}\right), e_{t}\right), \\
\tilde{\tilde{\tilde{h}}}_{r s}^{t}=\tilde{g}\left(h\left(J_{3} e_{r}, J_{3} e_{s}\right), e_{t}\right),
\end{gathered}
$$

with $i, j=\overline{1, n}, r, s, t=\overline{n+1, n+q}$.

Using the fact that $\sum_{1 \leq i<j \leq n} \sum_{r=n+1}^{n+q}\left(h_{i j}^{r}\right)^{2}$ is positive as a sum of squares, from (3.6), we get

$$
\begin{gathered}
\delta\left(\mathcal{D}^{\perp}\right)(x) \leq \frac{n(n+6 q+8)}{2} \cdot \frac{c}{4}+ \\
+\sum_{1 \leq i<j \leq n} \sum_{r=n+1}^{n+q} h_{i i}^{r} h_{j j}^{r}+\sum_{i=1}^{n} \sum_{r, s=n+1}^{n+q} h_{i i}^{r}\left[\tilde{h}_{s s}^{r}+\tilde{\tilde{h}}_{s s}^{r}+\tilde{\tilde{\tilde{h}}}_{s s}^{r}\right] .
\end{gathered}
$$

We consider the following quadratic forms $f_{t}: \mathbf{R}^{n+3 q} \rightarrow \mathbf{R}$,

$$
\begin{gathered}
f_{t}\left(h_{11}^{t}, \ldots, h_{n n}^{t} ; \tilde{h}_{n+1 ; n+1}^{t}, \ldots, \tilde{h}_{n+q ; n+q}^{t} ; \tilde{\tilde{h}}_{n+1 ; n+1}^{t}, \ldots, \tilde{\tilde{h}}_{n+q ; n+q}^{t} ; \tilde{\tilde{\tilde{h}}}_{n+1 ; n+1}^{t}, \ldots, \tilde{\tilde{\tilde{h}}}_{n+q ; n+q}^{t}\right)= \\
=\sum_{1 \leq i<j \leq n} h_{i i}^{t} h_{j j}^{t}+\sum_{i=1}^{n} \sum_{r=n+1}^{n+q} h_{i i}^{t}\left[\tilde{h}_{r r}^{t}+\tilde{\tilde{h}}_{r r}^{t}+\tilde{\tilde{h}}_{r r}^{t}\right], t=\overline{n+1, n+q} .
\end{gathered}
$$


For $f_{t}\left(h_{11}^{t}, \ldots, \tilde{\tilde{h}}_{n+q ; n+q}^{t}\right)$ we must find an upper bound, subject to

$$
\begin{gathered}
P: h_{11}^{t}+\ldots+h_{n n}^{t}+\tilde{h}_{n+1 ; n+1}^{t}+\ldots+\tilde{h}_{n+q ; n+q}^{t}+\tilde{\tilde{h}}_{n+1 ; n+1}^{t}+\ldots+\tilde{\tilde{h}}_{n+q ; n+q}^{t}+ \\
+\tilde{\tilde{\tilde{h}}}_{n+1 ; n+1}^{t}+\ldots+\tilde{\tilde{\tilde{h}}}_{n+q ; n+q}^{t}=c^{t}
\end{gathered}
$$

where $c^{t}$ is a real constant.

For this, we calculate the partial derivatives of $f_{t}$ :

$$
\begin{gathered}
\frac{\partial f_{t}}{\partial h_{i i}^{t}}=\sum_{1 \leq j \leq n}^{j \neq i} h_{j j}^{t}+\sum_{r=n+1}^{n+q}\left(\tilde{h}_{r r}^{t}+\tilde{\tilde{h}}_{r r}^{t}+\tilde{\tilde{\tilde{h}}}_{r r}^{t}\right), i=\overline{1, n}, \\
\frac{\partial f_{t}}{\partial \tilde{h}_{s s}^{t}}=\frac{\partial f_{t}}{\partial \tilde{\tilde{h}}_{s s}^{t}}=\frac{\partial f_{t}}{\partial \tilde{\tilde{\tilde{h}}}_{s s}^{t}}=\sum_{i=1}^{n} h_{i i}^{t}, s=\overline{n+1, n+q} .
\end{gathered}
$$

In the standard frame of $\mathbf{R}^{n+3 q}$, the Hessian of $f_{t}$ has the matrix:

$$
\left(\begin{array}{cc}
A & B \\
B^{t} & C
\end{array}\right)
$$

where $B \in \mathcal{M}_{n, 3 q}(\mathbf{R})$, with all the elements equal to $1, C \in \mathcal{M}_{3 q}(\mathbf{R})$, with all the elements equals to 0 and $A$ is the matrix :

$$
A=\left(\begin{array}{ccccc}
0 & 1 & 1 & \ldots & 1 \\
1 & 0 & 1 & \ldots & 1 \\
\ldots & \ldots & \ldots & \ldots & . \\
1 & 1 & 1 & \ldots & 0
\end{array}\right), \quad A \in \mathcal{M}_{n}(\mathbf{R})
$$

We obtain

$$
\begin{gathered}
\beta(X, X)=2 \sum_{1 \leq i<j \leq n} X_{i} X_{j}+2 \sum_{i=1}^{n} \sum_{r=n+1}^{n+q} X_{i}\left(\tilde{X}_{r}+\tilde{\tilde{X}}_{r}+\tilde{\tilde{\tilde{X}}}_{r}\right)= \\
=\left[\sum_{i=1}^{n} X_{i}+\sum_{r=n+1}^{n+q}\left(\tilde{X}_{r}+\tilde{\tilde{X}}_{r}+\tilde{\tilde{\tilde{X}}}_{r}\right)\right]^{2}-\sum_{i+1}^{n}\left(X_{i}\right)^{2}-\sum_{r=n+1}^{n+q}\left[\left(\tilde{X}_{r}\right)^{2}+\left(\tilde{\tilde{X}}_{r}\right)^{2}+\left(\tilde{\tilde{X}}_{r}\right)^{2}\right]- \\
-2 \sum_{r, s=n+1}^{n+q}\left(\tilde{X}_{r} \tilde{\tilde{X}}_{s}+\tilde{X}_{r} \tilde{\tilde{X}}_{s}+\tilde{\tilde{X}}_{r} \tilde{\tilde{X}}_{s}\right)= \\
=\left[\sum_{i=1}^{n} X_{i}+\sum_{r=n+1}^{n+q}\left(\tilde{X}_{r}+\tilde{\tilde{X}}_{r}+\tilde{\tilde{X}}_{r}\right)\right]^{2}-\sum_{i+1}^{n}\left(X_{i}\right)^{2}-\left[\sum_{n+1}^{n+q}\left(\tilde{X}_{r}+\tilde{\tilde{X}}_{r}+\tilde{\tilde{X}}_{r}\right)\right]^{2}= \\
=-\sum_{i+1}^{n}\left(X_{i}\right)^{2}-\left[\sum_{n+1}^{n+q}\left(\tilde{X}_{r}+\tilde{\tilde{X}}_{r}+\tilde{\tilde{\tilde{X}}}_{r}\right)\right]^{2}<0
\end{gathered}
$$

because $\left[\sum_{i=1}^{n} X_{i}+\sum_{r=n+1}^{n+q}\left(\tilde{X}_{r}+\tilde{\tilde{X}}_{r}+\tilde{\tilde{X}}_{r}\right)\right]^{2}=0, P$ being totally geodesic in $\mathbb{R}^{n+3 q}$. Then the Hessian of $f_{t}$ is negative semidefinite, so $f_{t}$ reaches its maximum (see Remark 2.2.).

Searching for the critical points $\left(h_{11}^{t}, \ldots, \tilde{\tilde{\tilde{h}}}_{n+q ; n+q}^{t}\right)$ of $f_{t}$, we find:

$$
\begin{gathered}
\frac{\partial f_{t}}{\partial h_{11}^{t}}=\frac{\partial f_{t}}{\partial h_{22}^{t}} \Longrightarrow \\
\left.\sum_{j=2}^{n} h_{j j}^{t}+\sum_{r=n+1}^{n+q}\left(\tilde{h}_{r r}^{t}+\tilde{\tilde{h}}_{r r}^{t}+\tilde{\tilde{\tilde{h}}}_{r r}^{t}\right)\right)=\sum_{1 \leq j \leq n}^{j \neq 2} h_{j j}^{t}+\sum_{r=n+1}^{n+q}\left(\tilde{h}_{r r}^{t}+\tilde{\tilde{h}}_{r r}^{t}+\tilde{\tilde{\tilde{h}}}_{r r}^{t}\right),
\end{gathered}
$$


which gives

$$
h_{11}^{t}=h_{22}^{t}=\ldots=h_{n n}^{t}=\lambda .
$$

Also

$$
\begin{gathered}
\frac{\partial f_{t}}{\partial h_{11}^{t}}=\frac{\partial f_{t}}{\partial \tilde{h}_{n+1 ; n+1}^{t}} \Longrightarrow \\
h_{11}^{t}=\sum_{r=n+1}^{n+q}\left(\tilde{h}_{r r}^{t}+\tilde{\tilde{h}}_{r r}^{t}+\tilde{\tilde{h}}_{r r}^{t}\right)=\lambda .
\end{gathered}
$$

From (3.10), (3.12) and (3.13) we obtain

$$
n \lambda+\lambda=c^{t} \Longrightarrow \lambda=\frac{c^{t}}{n+1}
$$

which gives

$$
h_{11}^{t}=h_{22}^{t}=\ldots=h_{n n}^{t}=\sum_{r=n+1}^{n+q}\left(\tilde{h}_{r r}^{t}+\tilde{\tilde{h}}_{r r}^{t}+\tilde{\tilde{h}}_{r r}^{t}\right)=\frac{c^{t}}{n+1} .
$$

Using the relations (3.14) in the expression of $f_{t}$ from (3.9) we have

$$
\begin{aligned}
& f_{t}\left(h_{11}^{t}, \ldots, \tilde{\tilde{\tilde{h}}}_{n+q ; n+q}^{t} \leq \frac{n(n-1)}{2} \cdot\left(\frac{c^{t}}{n+1}\right)^{2}+n \cdot\left(\frac{c^{t}}{n+1}\right)^{2}=\right. \\
& =\left(\frac{c^{t}}{n+1}\right)^{2} \cdot\left[\frac{n(n-1)}{2}+n\right]=\left(\frac{c^{t}}{n+1}\right)^{2} \cdot\left[\frac{n^{2}-n+2 n}{2}\right]= \\
& =\left(\frac{c^{t}}{n+1}\right)^{2} \cdot\left(\frac{n^{2}+n}{2}\right)=\left(\frac{c^{t}}{n+1}\right)^{2} \cdot \frac{n(n+1)}{2}=\frac{\left(c^{t}\right)^{2}}{2} \cdot \frac{n}{n+1},
\end{aligned}
$$

and then

$$
f_{t} \leq \frac{n}{2(n+1)} \cdot(n+3 q)^{2} \cdot\left\|H^{t}\right\|^{2}
$$

where

$$
H^{t}=\frac{1}{n+3 q} \cdot\left[\sum_{i=1}^{n} h_{i i}^{t}+\sum_{r=n+1}^{n+q}\left(\tilde{h}_{r r}^{t}+\tilde{\tilde{h}}_{r r}^{t}+\tilde{\tilde{\tilde{h}}}_{r r}^{t}\right)\right] .
$$

From (3.8) and (3.15) we obtain the relation $\left(^{*}\right)$.

The relations (3.4), (3.6) and (3.12) imply the conditions for the equality case.

\section{References}

[1] Al-Solamy, F., Chen, B.-Y. and Deshmukh, S., Two optimal inequalities for anti-holomorphic submanifolds and their applications, Taiwanese J. Math. 18 (2014), no.1, 199-217.

[2] Bejancu, A., QR-submanifolds of quaternion Kaehler manifolds, Chinese J. Math. 14 (1986), no. 2, 81-94.

[3] Chen, B.-Y., CR-submanifolds of a Kaehler manifold, J. Diff. Geom. 16 (1981), 305-323.

[4] Chen, B.-Y., An optimal inequality for CR-warped products in complex space forms involving CR $\delta$-invariant, Internat. J. Math. 23 (2012), no. 3, 1250045 (17 pages).

[5] Macsim, G. and Mihai, A., An inequality on quaternionic CR-submanifolds, Ann. Univ. Ovidius Constanţa, XXVI (2018), no. 3, to appear.

[6] Nash, J. F., The imbedding problem for Riemannian manifolds, Ann. of Math. 63 (1956), 20-63.

[7] Oprea, T., Optimizations on Riemannian submanifolds, Analele Univ. Buc. LIV 1 (2005), 127-136.

[8] Şahin, B., On QR-submanifolds of a quaternionic space forms, Turkish J. Math. 25 (2001), 413-425. 


\section{Affiliations}

GABRIEL MACSIM

ADDRESS: University of Bucharest, Doctoral School of Mathematics, Academiei Str. 14, 010014 Bucharest, Romania.

E-MAIL: gabi_macsim@yahoo.com

ORCID ID : 0000-0003-3071-3338

Adela MiHai

AdDRESS: Technical University of Civil Engineering, Department of Mathematics and Computer Science, Lacul Tei Bvd. 122-124, 020396 Bucharest, Romania.

E-MAIL: adela.mihai@utcb.ro

ORCID ID : 0000-0003-2033-8394 